\title{
PARP1 activation directs RNA binding proteins to DNA damages to form PARG reversible compartments enriched in damaged DNA
}

\author{
Olga Lavrik \\ LBCE \\ Institute of Chemical Biology and \\ Fundamental Medicine SB RAS \\ Novosibirsk, Russia \\ Maria Sukhanova \\ LBCE \\ Institute of Chemical Biology and \\ Fundamental Medicin SB RAS \\ Novosibirsk, Russia
}

\author{
Anastasia Singatulina \\ LBCE \\ Institute of Chemical Biology and \\ Fundamental Medicine SB RAS \\ Novosibirsk, Russia \\ Konstantin Naumenko \\ LBCE \\ Institute of Chemical Biology and \\ Fundamental Medicine SB RAS \\ Novosibirsk, Russia
}

\author{
Loic Hamon \\ SABNP \\ INSERM U1204 \\ Université Paris-Saclay \\ Evry, France \\ David Pastré \\ SABNPINSERM U1204 \\ Université Paris-Saclay \\ Evry, France
}

\begin{abstract}
Poly(ADP-ribose) polymerase 1 (PARP1) synthesizes poly(ADP-ribose) (PAR) at DNA damage sites to recruit DNA repair factors. PAR plays a key role in regulation of multiple processes in high eukaryotic cells and interacts with DNA and RNA binding proteins. Among proteins relocated on damaged DNA, the nuclear RNA-binding protein FUS is one of the most abundant, raising the issue about its involvement in DNA repair. We reconstituted the PARP-1/PAR/damaged DNA and analyzed this system by atomic force microscopy in the presence of FUS. We demonstrated the dissociation of FUS from mRNA in the presence of PAR, its recruitment at DNA damage sites via its binding to PAR, and observed assembly of dynamic compartments in which damaged DNA is concentrated. FUS contains disordered prion-like domains and its interaction with PAR is a driving force in formation of membrane less compartments by liquid-liquid phase separation. The hydrolysis of PAR by poly(ADP-ribose) glycohydrolase (PARG) results in dissociation of damaged DNA-rich compartments and initiates the nucleocytoplasmic shuttling of FUS in cells. We anticipate that FUS facilitates DNA repair through the transient compartmentalization of DNA damage sites and concentration in these compartments of DNA repair proteins. This reversible process permits to select damaged DNA from undamaged one and accelerates DNA repair. Other RNA binding protein YB-1 interacts with PAR and stimulates PARP1 activity. Multifunctional protein YB-1 is located in cytoplasm but also shows its nuclear localization. The role of YB-1 in the formation of liquid compartments formed at DNA damages under PARP1 activation was investigated. Therefore, this study shed light on the role of PARP1 and RNA binding proteins containing prionlike domains in the formation of dynamic liquid compartments, which can facilitate DNA repair by concentrating in these structures damaged DNA and repair proteins.
\end{abstract}

Keywords - DNA repair; RNA-binding proteins; atomic force microscopy, cancer, liquid-liquid phase separation, neurodegenerative disease, poly(ADP-ribose), poly(ADP-ribose) polymerase 1

\section{Introduction}

In mammalian cells, DNA single- and double-strand breaks trigger a complex cascade of events in which the members of the poly(ADP-ribose)polymerase family such PARP1 recognize damaged DNA and synthesize poly(ADP-

The work was supported by RSF (project No. 19-04-00882),

RFBR (project No. 18-04-00882) and Genopole Evry. ribose) (PAR) polymer covalently attached to themselves or to other acceptor proteins [1]. The central enzyme for PAR production in cells and the main target of poly(ADPribosyl)ation (PARylation) during DNA damage is PARP1 [2]. PARP1-mediated PARylation of proteins and PAR polymer synthesis is one of the earliest events in DNA damage response. It facilitates the initial recruitment of proteins to DNA lesions and orchestrates a wave of chromatin remodelling events during the DNA repair. The protein PARylation is subjected to tight control by the enzymes that degrade PAR. The key ADP-ribose-degrading enzyme is poly(ADP-ribose)glycohydrolase (PARG), which makes protein PARylation a reversible post-translational modification [2]. The synthesis of PAR polymers in response to genotoxic stress provides a landing platform for a plethora of PAR-binding proteins among them number of RNAbinding proteins (RBPs) [3]. Both high PARylation level among RBPs and their abundance in nuclear regions damaged by short laser beam exposures raise issues about the putative role of the proteins in DNA damage response [4]. Here, we focus our attention on RBPs such as FUS (FUS/TLS, Fused in Sarcoma) and YB-1 (Y-box-binding protein 1). FUS is a multifunctional DNA/RNA-binding protein that is involved in the regulation of transcription, pre-mRNA splicing, mRNA transport and local mRNA translation, storage and can be implicated in DNA repair response. FUS is a member of the FET family and one of the most abundant and highly PARylated nuclear RNA-binding proteins. Similar to FUS, YB-1 carries out its functions in RNA methabolism and likely in DNA repair [3]. YB-1, as a transcription factor, controls the expression of stress-induced genes and the genes involved in DNA repair and as an RNA-binding protein, YB-1 mediates pre-mRNA splicing, is one of the major proteins constituting RNP granules in the cytoplasm, and modulates mRNA translation. It has been recently shown that PAR can nucleate the intracellular phase transitions of such RBPs such as FUS at microlaser-generated sites of DNA lesions [4]. Intracellular compartmentalization initiated by PAR-dependent phase separation can underlie the mechanisms by which PAR is involved in DNA- and RNA-dependent cellular events: for example, the formation of stress-granules, nucleoli, spliceosomes, and transcriptosomes [3]. The molecular mechanisms responsible for the formation and the possible functions of these compartments are, therefore, difficult to 
address in a cellular context. Further advances in the understanding of the function of FUS and YB-1 in DNA repair, notably when in interaction with PAR, are critically required. In the present work, we investigate in vitro and in vivo the FUS and YB-1 interaction with PAR to elucidate the mechanism involvement of these proteins in DNA repair through PARP1 activation.

\section{Results}

We developed an original approach based on a single molecule analysis by atomic force microscopy (AFM) to reconstitute the molecular system that serves to recruit FUS at DNA damage sites, including factors such as intact and damaged DNA, PARP1, and mRNA [5]. We also have applied the new real-time technique to explore YB-1-PARP1 interplay during the poly(ADP-ribosyl)ation process [6]. We have shown the local recruitment of FUS to PAR synthesized by PARP1 at damaged DNA sites and found that FUS then triggers the formation of large compartments in which damaged DNA is enriched. We have demonstrated the reversible nature of these compartments as the hydrolysis of PAR by PARG is sufficient to dissociate damaged DNA compartments formed by FUS. We also shown that YB-1 can stimulate PARP1 in the absence of magnesium, and that YB1-PARP1 interplay can be mediated and regulated not only by the DNA at the initial stage of poly(ADP-ribosyl)ation, but also by poly(ADP-ribose) during elongation stage of the polymer synthesis.

Using AFM-based single molecule visualization, we have analyzed FUS-PAR interactions in the reconstituted molecular system including mRNA to mimic nuclear mRNA targets of FUS, damaged DNA, PARP-1 (to recognize DNA damages sites), $\mathrm{NAD}^{+}$(to trigger the synthesis of PAR by PARP1), and PARG (to hydrolyze PAR). We have demonstrated not only the strong affinity of FUS for PAR, but the recruitment of FUS to PAR synthesized by PARP1 at DNA damage sites followed by the formation of large aggregates or compartments in which damaged DNA is concentrated (Fig. 1a). Howevev, YB-1 or two other mRNA-binding proteins (HuR, G3BP1) that also have low complexity domain and also bind to PAR but fail to trigger the formation of large DNA-rich compartments (data not shown). In the case of YB-1, we found that YB-1 can form heteromeric complex with PARP1 on damaged DNA, serving as preferable PAR acceptor at the initiation stage. During elongation unmodified YB-1 molecules appear to non-selectively bind growing polymers of poly(ADP-ribose) rather than DNA at the PARP1 boarding site (data not shown).

\section{Conclusions}

Our data suggest that transient molecular assemblies triggered by FUS upon PARP-1 activation may facilitate DNA repair through compartmentalization of DNA damage sites. The hydrolysis of these compartments with PARG provides reversibility of the whole process by dissociation of these compartments (Fig. 1b).

In the case of YB-1, non-covalent binding of unstructured positively charged C-tail of YB-1 to PAR polymers during auto-modification of PARP1 stabilizes the catalytically active PARP1-DNA complex and stimulates PAR elongation.

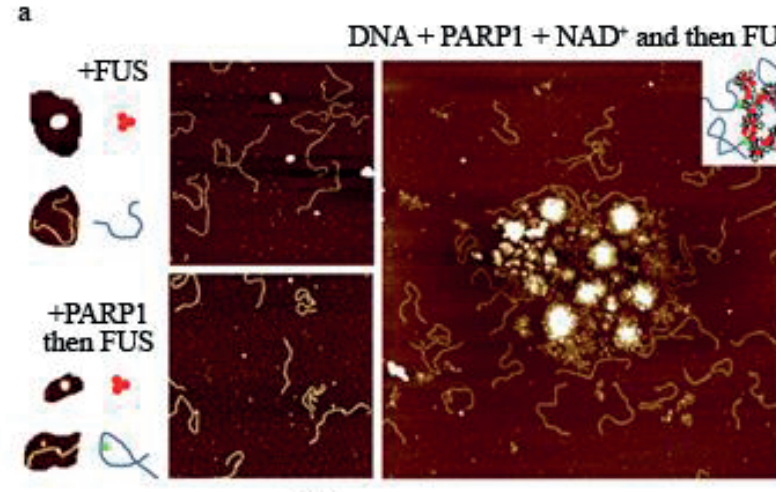

$\overline{200 \mathrm{~nm}}$

b

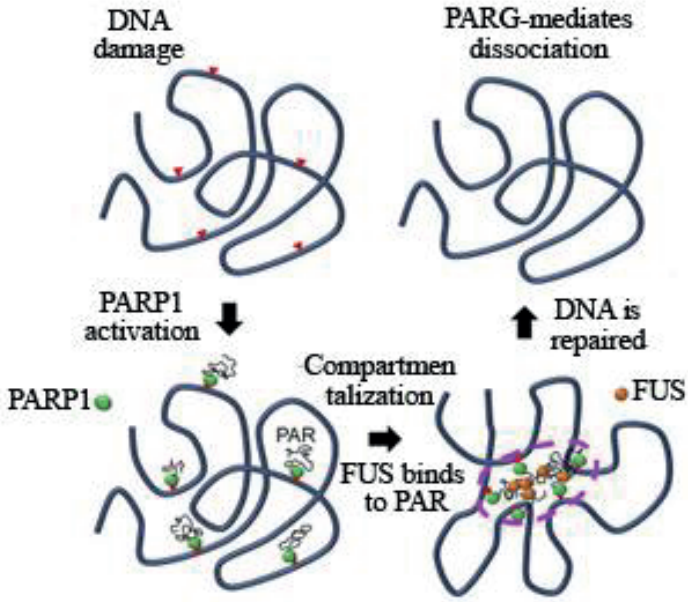

PARG-mediates dissociation

Fig. 1. FUS forms DNA-rich compartments upon PARP1 activation.a. AFM images of DNA after incubation with PARP1 in the presence of $\mathrm{NAD}^{+}$to trigger the synthesis of PAR, followed by the addition of FUS. b. Schematic view of the different steps of damaged DNA compartmentalization by FUS.

It is possible that regions of low complexity or prione-like domain in FUS (or YB-1) plays primary role in modulation of interaction of both protein with PAR.

\section{REFERENCES}

[1] E. E. Alemasova, and O. I. Lavrik, "Poly (ADP-ribosyl) ation by PARP1: reaction mechanism and regulatory proteins" Nucleic Acids Res., vol. 47, pp. 3811-3827, May 2019.

[2] D. d'Amours, S. Desnoyers, I. d'Silva, and G.G. Poirier, "Poly(ADPribosyl)ation reactions in the regulation of nuclear functions", Biochem. J., vol. 342, pp. 249-268, September 1999.

[3] E. E. Alemasova, and O. I. Lavrik, "At the interface of three nucleic acids: the role of RNA-binding proteins and poly (ADP-ribose) in DNA repair", Acta Naturae, vol. 9, pp. 4-16, April 2017.

[4] S. L. Rulten, A. Rotheray, R. L. Green, G. J. Grundy, D. A. Moore, F. Gomez-Herreros, and K. W. Caldecott, "PARP-1 dependent recruitment of the amyotrophic lateral sclerosis-associated protein FUS/TLS to sites of oxidative DNA damage", Nucleic Acids Res, vol. 42, pp. 307-314, January 2014.

[5] A. S. Singatulina, L. Hamon, M. V. Sukhanova, B. Desforges, V. Joshi, A. Bouhss, O. I. Lavrik, and D. Pastré, "PARP-1 activation directs FUS to DNA damage sites to form PARG-reversible compartments enriched in damaged DNA" Cell Rep., vol. 27, pp. 1809-1821, May 2019.

[6] E. E. Alemasova, K. N. Naumenko, T. A. Kurgina, R. O. Anarbaev, and O. I. Lavrik, "The multifunctional protein YB-1 potentiates PARP1 activity and decreases the efficiency of PARP1 inhibitors", Oncotarget, vol. 9, pp. 23349-23365, May 2018. 